CERN-TH.98-226

DFPD-98/TH-34

\title{
Neutrino Mass Textures from Oscillations with Maximal Mixing
}

\author{
Guido Altarelli \\ Theoretical Physics Division, CERN \\ CH - 1211 Geneva 23 \\ and \\ Università di Roma Tre, Rome, Italy \\ Ferruccio Feruglio \\ Università di Padova, Padova, Italy \\ and \\ I.N.F.N., Sezione di Padova
}

\begin{abstract}
We study the implications of neutrino oscillations with maximal mixing for the neutrino Dirac and Majorana matrices in the see-saw mechanism for three non degenerate neutrino masses. We find the form of the Dirac matrix for a structure-less Majorana matrix and, conversely, the structure of the Majorana matrix if the Dirac matrix is according to our naive intuition. We give some examples of Majorana matrices that, in a $3 \times 3$ context, lead to maximal mixing without too much fine tuning and cross talk with the Dirac input.
\end{abstract}

CERN-TH.98-226

DFPD-98/TH-34

July 1998 
Recent data from Superkamiokande [1](and also MACRO [2]) have provided a more solid experimental basis for neutrino oscillations as an explanation of the atmospheric neutrino anomaly. In addition, the solar neutrino deficit is also probably an indication of a different sort of neutrino oscillations. Results from the laboratory experiment by the LNSD collaboration [3] can also be considered as a possible indication of yet another type of neutrino oscillation. But the preliminary data from Karmen [4 have failed to reproduce this evidence. The case of LNSD oscillations is far from closed but one can tentatively assume, pending the results of continuing experiments, that the signal will not persist. Then solar and atmospheric neutrino oscillations can possibly be explained in terms of the three known flavours of neutrinos without invoking extra sterile species. Neutrino oscillations for atmospheric neutrinos require $\nu_{\mu} \rightarrow \nu_{\tau}$ with $\Delta m_{\text {atm }}^{2} \sim 2 \cdot 10^{-3} \mathrm{eV}^{2}$ and a nearly maximal mixing angle $\sin ^{2} 2 \theta_{\text {atm }} \geq 0.8$. Furthermore the last results from Superkamiokande allow [6] for a solution of the solar neutrino deficit in terms of $\nu_{e}$ disappearance vacuum oscillations (as opposed to MSW [5] oscillations within the sun) with $\Delta m_{\text {sol }}^{2} \sim 10^{-10} \mathrm{eV}^{2}$ and again nearly maximal mixing angles. Among the large and small angle MSW solutions the small angle one (with [6] $\Delta m_{\text {sol }}^{2} \sim 0.5 \cdot 10^{-5} \mathrm{eV}^{2}$ and $\sin ^{2} 2 \theta_{\text {sol }} \sim 5.5 \cdot 10^{-3}$ ) is more likely at the moment than the large angle MSW solution. Of course experimental uncertainties are still large and the numbers given here are merely indicative. Thus atmospheric neutrinos, if a genuine signal of oscillations, certainly require nearly maximal mixing and possibly also solar neutrinos may arise from nearly maximal mixing. Large mixings are very interesting because a first guess was in favour of small mixings in the neutrino sector in analogy to what is observed for quarks. If confirmed, single or double maximal mixings can provide an important hint on the mechanisms that generate neutrino masses.

In the present note we start from the simplest (at least to our taste) scenario with only three flavours of neutrinos that receive masses from the see-saw mechanism, with a given hierarchical structure for the neutrino mass eigenvalues $m_{i}$ (e.g. $m_{3} \gg m_{2} \gg m_{1}$ or $m_{3} \gg m_{2} \sim m_{1}$ ). We consider the simplest version of the see-saw mechanism with one Dirac, $m_{D}$, and one Majorana, $M$, mass matrix, related to the neutrino mass matrix $m_{\nu}$, in the basis where the charged lepton mass matrix is diagonal, by

$$
m_{\nu}=m_{D}^{T} M^{-1} m_{D}
$$

As well known this is not the most general see-saw mechanism because we are not including the left-left Majorana mass block. This assumption is done here for reasons of simplicity and because it represents the most constrained situation: allowing this extra matrix would leave more freedom. We study the implications of maximal mixing (either single or double) on the Dirac and Majorana matrix structure. One may imagine that maximal mixing cannot be accommodated in a natural way in presence of a large spread of the neutrino masses and that maximal mixing goes in the direction of degenerate masses. We want to investigate to what extent this statement is really justified. We analyse in detail the most interesting limiting cases. In one (sect.2) all the structure arises from the Dirac matrix $m_{D}$ while $M$ is the simplest. We find that the resulting Dirac matrix has a well defined structure. Once this structure is realized then maximal mixing is obtained without fine tuning. Or, alternatively (sect.3), the Dirac matrix is taken according to the intuition that it should be similar to the up quark mass 
matrix, (for example, with small mixings among mass splitted states, possibly with a remnant of the top, charm, up mass pattern) and the large mixing(s) are induced by $M$. For non degenerate masses what is obtained is a texture of the Majorana matrix $M_{i j}$ with increasing absolute values when $\mathrm{i}+\mathrm{j}$ increases, which is more pronounced for double versus single maximal mixing. However in this case the realization of the specific texture is not sufficient and extra fine tuning is needed. More interesting solutions are found if we allow near degeneracy for $m_{1}$ and $m_{2}$.

Our main framework is the most obvious case where the observed relation $\Delta m_{\text {atm }}^{2} \gg \Delta m_{\text {sol }}^{2}$ is a reflection of the mass hierarchy $m_{3} \gg m_{2,1}$, with no prejudice on the $m_{1}, m_{2}$ relation. Maximal atmospheric neutrino mixing and the requirement that the electron neutrino does not participate in the atmospheric oscillations, as indicated by the Superkamiokande [1] and Chooz [7] data, lead directly to the following structure of the $U_{f i}(\mathrm{f}=\mathrm{e}, \mu, \tau, \mathrm{i}=1,2,3)$ real orthogonal mixing matrix, apart from sign convention redefinitions (here we are not interested in $\mathrm{CP}$ violation effects, which are small in our context, being exactly zero for $U_{e 3}=0$ ):

$$
U_{f i}=\left[\begin{array}{ccc}
c & -s & 0 \\
s / \sqrt{2} & c / \sqrt{2} & -1 / \sqrt{2} \\
s / \sqrt{2} & c / \sqrt{2} & +1 / \sqrt{2}
\end{array}\right]
$$

This result is obtained by a simple generalization of the analysis of ref. [8] to the case of arbitrary solar mixing angle 円 $\left(s \equiv \sin \theta_{\text {sun }}, c \equiv \cos \theta_{\text {sun }}\right): c=s=1 / \sqrt{2}$ for maximal solar mixing, while $\sin ^{2} 2 \theta_{\text {sun }} \sim 4 s^{2} \sim 5.5 \cdot 10^{-3}$ for the small angle MSW solution. The vanishing of $U_{e 3}$ guarantees that $\nu_{e}$ does not participate in the atmospheric oscillations and the relation $\left|U_{\mu 3}\right|=\left|U_{\tau 3}\right|=1 / \sqrt{2}$ implies maximal mixing for atmospheric neutrinos. The non diagonal oscillation probabilities are

$$
\begin{aligned}
P\left(\nu_{e} \leftrightarrow \nu_{\mu}\right)= & P\left(\nu_{e} \leftrightarrow \nu_{\tau}\right)=2 c^{2} s^{2} \sin ^{2} \Delta_{\text {sun }} \\
P\left(\nu_{\mu} \leftrightarrow \nu_{\tau}\right) & =\sin ^{2} \Delta_{a t m}-c^{2} s^{2} \sin ^{2} \Delta_{\text {sun }}
\end{aligned}
$$

Note that we are assuming only two frequencies, given by $\Delta_{\text {sun }} \propto m_{2}^{2}-m_{1}^{2}$ and $\Delta_{a t m} \propto m_{3}^{2}-m_{1,2}^{2}$. A more general analysis can be found in ref. [10]. The neutrino mass matrix is given by $U m_{\text {diag }} U^{T}$ with $m_{\text {diag }}=\operatorname{Diag}\left[m_{1}, m_{2}, m_{3}\right]$. In the following we will always give $m_{\nu}$ in units of $m_{3} / 2, m_{D}$ in units of $m_{0} / \sqrt{2}$, and $M$ in units of some large mass $\bar{M}$ related to $m_{3}$ and $m_{0}$ by $m_{3}=m_{0}^{2} / \bar{M}$. With this convention, for generic $s$ one finds

$$
m_{\nu}=\left[\begin{array}{ccc}
2 \epsilon & \delta & \delta \\
\delta & 1+\epsilon_{2} & -1+\epsilon_{2} \\
\delta & -1+\epsilon_{2} & 1+\epsilon_{2}
\end{array}\right]
$$

with

$$
\epsilon=\left(\mu_{1} c^{2}+\mu_{2} s^{2}\right), \quad \delta=\sqrt{2}\left(\mu_{1}-\mu_{2}\right) c s, \quad \epsilon_{2}=\left(\mu_{1} s^{2}+\mu_{2} c^{2}\right)
$$

where $\mu_{i} \equiv m_{i} / m_{3}$. We see that the existence of one maximal mixing and $U_{e 3}=0$ are the most important input that leads to the matrix form in eq. (4). The value of the solar neutrino mixing angle can be left free.

\footnotetext{
${ }^{1}$ An analogous parametrization has been discussed in ref. [9].
} 
We consider first the case of the simplest Majorana matrix $M$, namely a multiple of the identity: $M \equiv 1$. In the non degenerate case $m_{3} \gg m_{2}, m_{1}$ we find that eq. (II) is satisfied by the following texture:

$$
m_{D}=\left[\begin{array}{ccc}
\lambda & \lambda & \lambda \\
\lambda & \sim c & \sim-c \\
\lambda & \sim-t & \sim t
\end{array}\right] \quad, \quad c^{2}+t^{2}=1
$$

where $\lambda$ is a small parameter related to $\mu_{1}, \mu_{2}$ and $s$. By $\sim c(t)$ we mean $c(t)+O\left(\lambda^{n}\right)$ and by $\lambda$ we mean $O\left(\lambda^{n}\right)$ with $n \geq 1$ f. We have obtained this texture starting from a Majorana matrix which is a multiple of the identity. But a particularly remarkable feature of this texture is that it satisfies eq.(11) for whatever symmetric non singular Majorana matrix with all matrix elements of order 1. Precisely, if we denote by $m_{D}^{0}$ the matrix obtained by setting $\lambda=0$ in eq.(可), then, up to a multiplicative factor, $m_{D}^{0}$ provides an exact solution of eq. (1) for any symmetric non singular Majorana matrix. This feature arises because the diagonal form of $m_{D}^{0}$ has only the entry 33 different from zero and hence is proportional to a projector. For $M=1$ an example of double maximal mixing is given by the matrix

$$
m_{D}=1 / \sqrt{2}\left[\begin{array}{ccc}
2 \lambda & \lambda & \lambda \\
\lambda & 1+\lambda & -1+\lambda \\
\lambda & -1+\lambda & 1+\lambda
\end{array}\right]
$$

with $\lambda^{2} \sim 10^{-4}-10^{-5}$. Note that an attractive feature of double maximal mixing is that for $m_{3} \gg m_{1,2}, \mu_{2}$ comes remarkably close to $m_{c}^{2} / m_{t}^{2}$. Also note that in general, if we set $m_{3} \sim m_{t}^{2} /(\bar{M})$, then $\bar{M}$ is close to the unification mass $m_{G U T}$. Thus presumably $\lambda^{2}$ could be related to $m_{c}^{2} / m_{t}^{2}$. Similarly, an example of single maximal mixing is given by the matrix

$$
m_{D}=1 / \sqrt{2}\left[\begin{array}{ccc}
0 & \lambda^{2} & \lambda^{2} \\
\lambda^{2} & 1+\lambda & -1+\lambda \\
\lambda^{2} & -1+\lambda & 1+\lambda
\end{array}\right]
$$

with $\lambda^{2} \sim 10^{-3}$. The pattern of eq. (6) can be also specialized to the case $t \gg c$. For instance, for $M=1$ a double maximal mixing solution is offered by the matrix

$$
m_{D}=\left[\begin{array}{ccc}
\lambda^{2} & \lambda & 0 \\
\lambda & \lambda & \lambda \\
0 & -1 & 1
\end{array}\right]
$$

with $\lambda^{2} \sim 10^{-4}$. A further example of single maximal mixing is provided by the matrix:

$$
m_{D}=\left[\begin{array}{ccc}
\lambda^{2} & \lambda^{2} & 0 \\
\lambda^{2} & \lambda & \lambda \\
0 & -1 & 1
\end{array}\right]
$$

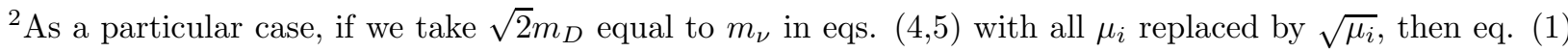
is identically satisfied.
} 
with $\lambda^{2} \sim 10^{-2}$. In all the above examples, eqs. (7-10), we have complete non degeneracy $m_{3}^{2} \gg m_{2}^{2} \gg m_{1}^{2}$.

We thus conclude that in the non degenerate case to explain atmospheric neutrinos in terms of $\nu_{\mu} \leftrightarrow \nu_{\tau}$ oscillations with maximal mixing, for a non special Majorana matrix, one needs a Dirac mass matrix with the texture in eq.(6). This texture is independent of the amount of mixing for solar neutrinos. Thus if a natural way of generating the texture in eq. (6) is found the problem is solved. Examples of strategies to approach this problem are given in refs. [11, 12]. But if one insists that large mixings are excluded in the Dirac sector then one can try to introduce a special texture in the Majorana sector. The required Majorana texture is discussed in the next section.

\section{3}

We want to determine the Majorana mass matrix for the simplest and most intuitive configuration for the Dirac matrix. So we take $m_{D}$ close to diagonal and given by

$$
m_{D}=\left[\begin{array}{lll}
u & 0 & 0 \\
0 & c & d \\
0 & f & t
\end{array}\right]
$$

where the zeroes represent smaller entries. For the time being the magnitudes of $u, c$ and $t$ are not specified but the notation is suggestive of up, charm and top quarks. For $f=d$ the general form of $M$ that reproduces $m_{\nu}$ in eqs.(田5) via eq.(11) is given by

$M=1 / D\left[\begin{array}{ccc}\epsilon_{2} u^{2} & -\frac{\delta}{2} u(c+d) & -\frac{\delta}{2} u(t+d) \\ -\frac{\delta}{2} u(c+d) & \frac{D}{4}(c-d)^{2}+\frac{\epsilon}{2}(c+d)^{2} & -\frac{D}{4}(c-d)(t-d)+\frac{\epsilon}{2}(c+d)(t+d) \\ -\frac{\delta}{2} u(t+d) & -\frac{D}{4}(c-d)(t-d)+\frac{\epsilon}{2}(c+d)(t+d) & \frac{D}{4}(t-d)^{2}+\frac{\epsilon}{2}(t+d)^{2}\end{array}\right]$

where

$$
D=\frac{\operatorname{Det}\left[m_{\nu}\right]}{4}=2 \epsilon \epsilon_{2}-\delta^{2}=2 \mu_{1} \mu_{2}
$$

Note that $\operatorname{Det}[M]=\left(\operatorname{Det}\left[m_{D}\right]\right)^{2} / \operatorname{Det}\left[m_{\nu}\right]=\left[u\left(c t-d^{2}\right)\right]^{2} /\left(8 \mu_{1} \mu_{2}\right)$. If the normalizing factor $\bar{M}$ is appropriately chosen, without loss of generality, we can take $\operatorname{Det}[M]$ of order 1 , and then eq.(13) is an important constraint on the relative magnitudes of $u, c, d(=f)$ and $t$ vs $\mu_{1}$ and $\mu_{2}$.

We see immediately from eq.(12) that, for $t \gg c, u$ and $\epsilon \sim \epsilon_{2} \sim \delta$ small, $D$ is in general negligible and the matrix elements $M_{i j}$ tend to increase when $i+j$ increases. For example, consider the case of double maximal mixing (vacuum solution for solar neutrinos). The gap between $\Delta m_{\text {atm }}^{2}$ and $\Delta m_{\text {sol }}^{2}$ is particularly large in this case and suggests a pronounced hierarchy of neutrino masses. If we take $\mu_{2} \sim \lambda^{2}, \mu_{1} \sim \lambda^{4}$ and correspondingly $u \sim \lambda^{2}, c, d(=f) \sim \lambda$ and 
$t \sim 1$, then we have $\epsilon \sim \epsilon_{2} \sim \delta \sim \lambda^{2}$ and $D \sim \lambda^{6}$. The resulting texture for $M$ is given by

$$
M=\left[\begin{array}{ccc}
1 & \frac{1}{\lambda} & \frac{1}{\lambda^{2}} \\
\frac{1}{\lambda} & \frac{1}{\lambda^{2}} & \frac{1}{\lambda^{3}} \\
\frac{1}{\lambda^{2}} & \frac{1}{\lambda^{3}} & \frac{1}{\lambda^{4}}
\end{array}\right]
$$

If we instead take $\mu_{2} \sim \mu_{1} \sim \lambda^{2}$ and $u \sim c \sim \lambda$ the entries in $M$ are less singular, i.e. the pattern is attenuated.

In the case of the small MSW solar neutrino solution, $\Delta m_{\text {sol }}^{2}$ is larger by about 5 orders of magnitude, thus for $m_{3} \gg m_{2} \gg m_{1}, \mu_{2}$ is larger by about 2.5 orders of magnitude and $\lambda$ is a factor of $\sim 20$ larger. Also $\delta$ is suppressed by the small mixing angle $s$, and numerically $s \sim \lambda^{2}$. In this case $\epsilon \sim \mu_{1}^{2}$ could be smaller than $\epsilon_{2} \sim \mu_{2}^{2}$. These features also result in an attenuation of the texture. If we take $\mu_{2} \sim \lambda^{2}, \mu_{1} \sim \lambda^{4}$ and correspondingly $u \sim \lambda^{2}, c, d(=f) \sim \lambda$ and $t \sim 1$, then we can now have $\epsilon \sim \lambda^{4}, \epsilon_{2} \sim \lambda^{2}, \delta \sim \lambda^{4}$ and $D \sim \lambda^{6}$. The resulting texture for $M$ is given by

$$
M=\left[\begin{array}{ccc}
1 & \lambda & 1 \\
\lambda & 1 & \frac{1}{\lambda} \\
1 & \frac{1}{\lambda} & \frac{1}{\lambda^{2}}
\end{array}\right]
$$

If we instead take $\mu_{2} \sim \mu_{1} \sim \lambda^{2}$ and $u \sim c \sim \lambda$ with $\epsilon \sim \epsilon_{2} \sim \lambda^{2}$ and $\delta \sim \lambda^{4}$ one finds that the texture is further attenuated.

The essential difference with the Dirac case is that, in the Majorana case, while the above type of textures are what comes out in absence of special relations among the coefficients, they are not sufficient. The ratios of the different entries have to be tuned in the way shown by the general expression in eq.(12), not only in order to obtain a determinant of order 1, but also to ensure the additional delicate cancellations needed to lead to the correct mixings. In other words, the nearly diagonal Dirac matrix and the Majorana matrix must together conspire in order to produce the result. Some crosstalk between the two matrices can be induced by the diagonalization of the charged lepton mass matrix (that changes $M^{-1} \rightarrow V M^{-1} V^{T}$ ). But in the logic of small Dirac mixing this interconnection should be limited to small terms.

The only possibility for a relatively disconnected first approximation is to go to some limiting form for the Majorana matrix, obtained by playing with the parameters $\epsilon, \epsilon_{2}$ and $\delta$. We find that this is only possible if $\mu_{1}$ and $\mu_{2}$ are nearly degenerate, because then $\delta$ can be of different order than the $\epsilon$ 's. We want a texture for $M$ of $\mathrm{O}(1)$ and $\mathrm{O}(0)$ entries, as much as possible independent of the $m_{D}$ matrix elements, that in conjunction with $m_{D}$ given in eq.(11) produce large mixing(s). Solutions for the Majorana matrix can be obtained by discussing the limiting case where the small quantities $\epsilon, \epsilon_{2}, \delta$ vanish. Then, eq. (ID) represents a set of constraints on the most general symmetric and invertible matrix $M$.

For instance, at first we demand small non diagonal terms in the Dirac matrix. By taking in eq. (11) $c=t=1$ and by setting, in first approximation, $u=d=f=0$ (i.e. $m_{D}=$ $\operatorname{Diag}[0,1,1])$, we obtain the result:

$$
M=\left[\begin{array}{ccc}
0 & C & C \\
C & 2 A-B+1 & A \\
C & A & B
\end{array}\right]
$$


Here $\mathrm{A}, \mathrm{B}$ and $\mathrm{C}(\neq 0)$ are independent parameters. At this point we can turn on small entries in the Dirac matrix. For instance, if we take $A, B, C \sim O(1), u \sim \lambda, d \sim f \sim k \lambda$, by small readjustments and by taking $\lambda$ close to $m_{c} / m_{t}$ we can find an acceptable doubly mixed solution with $m_{3}$ large and $\mu_{1}$ and $\mu_{2}$ nearly degenerate. This recipe is somewhat similar to the one proposed in a $2 \times 2$ context in ref. [13.

Alternatively we can try to restore the hierarchy $t \gg c$, by taking $m_{D}$ symmetric but non diagonal. In fact, for $d=f=t=1$ and $u=c=0$, we obtain the result

$$
M=\left[\begin{array}{ccc}
0 & C & 2 C \\
C & B & A \\
2 C & A & 4 A-4 B+1
\end{array}\right]
$$

where $A, B$ and $C(\neq 0)$ are independent quantities. Again, for $c \sim \lambda$ and $u$ small, this corresponds to double maximal mixing.

An additional class of solutions with the Dirac matrix quasi diagonal and hierarchical can be obtained starting from a non symmetric form of $m_{D}: d=-c$ and $f$ generic in eq. (11). For $u=0$, we find that

$$
M=\left[\begin{array}{lll}
0 & 0 & C \\
0 & D & A \\
C & A & B
\end{array}\right]
$$

solves eq. (1) up to the overall factor $c^{2} / D$ that can be absorbed in the definitions of $m_{0}$ and $\bar{M}$. By considering a small non-vanishing $u, m_{\nu}$ acquires 12 and 13 entries of order $u / c^{2}$ compared to the entries in the 2-3 block. A remarkable feature of this solution is that all what is needed in order to reproduce the desired pattern is to set to zero three elements of $M$, as displayed in eq. (18). The remaining quantities $A, B, C(\neq 0)$ and $D(\neq 0)$ are completely independent from the Dirac data. By taking $A, B, C, D, t$ of $O(1), f \sim \sqrt{\lambda}, c(=-d) \sim \lambda$ and $u \sim \lambda^{3}$ with $\lambda \sim 10^{-2}$ we find an acceptable solution with double maximal mixing. A particular case $A=B=0$ of this pattern has been considered in ref. [14]. For the last two cases, eqs. (17) and (18), the ratio $m_{2} / m_{3}$ is in general not related to $c / t$ although numerically it can be tuned to be so.

It is interesting that we find that double maximal mixing is simpler to obtain in this framework (because both $\epsilon$ and $\epsilon_{2}$ are smaller than $\delta$ and their difference does not appear). On the other hand we do not accept to fine tune $s$ in eqs.(5) in terms of $\mu_{1}$ and $\mu_{2}$ in order to make one $\epsilon$ of different order than the other. We also observe that, by appropriately choosing the parameters in eqs. (16), (17) and (18), one can obtain special Majorana textures with up to six vanishing entries.

\section{4}

In conclusion, we have studied the implications of neutrino oscillations with maximal mixing for the neutrino Dirac and Majorana matrices in the see-saw mechanism. Maximal mixing 
can be taken as an indication of nearly degenerate neutrino masses, because in perturbation theory mixing is small among widely non degenerate states. For the widely spread quark mass eigenstates the mixings are indeed small. In fact nearly degenerate neutrino masses have been widely considered recently. One advantage of full near degeneracy is that the three neutrinos can still provide a component of hot dark matter if the common mass is around $1 \mathrm{eV}$. We think that it is not really clear at the moment that a hot dark matter component is really needed [15]. So we have studied the non degenerate case $m_{3}^{2} \gg m_{1,2}^{2}$ and we have determined the conditions for maximal mixing to be imposed to the Dirac and the Majorana matrices of the see-saw mechanism. For a non special Majorana matrix the Dirac matrix structure is simple and well defined. Opposite $\mathrm{j} 2$ and $\mathrm{j} 3(j=2,3)$ matrix elements are required in the $\nu_{\mu}$ - $\nu_{\tau}$ mass entries and small matrix elements elsewhere. But once this requirement is met no additional fine tuning is needed. There are examples of strategies that may lead to this pattern in the context of grand unified theories [12]. So we do not consider that this possibility is to be discarded. On the contrary even if the Dirac matrix does not involve large mixings, the latter can still be obtained through the Majorana matrix. We have exhibited some examples of Majorana textures that lead to double maximal mixing without too much fine tuning and cross talk with the Dirac input. Further work is clearly needed to generate in a natural way the proposed textures from some reasonable dynamical context.

\section{References}

[1] Y. Fukuda et al., hep-ex/9805006, hep-ex/9805021 and hep-ph/9807003.

[2] M. Ambrosio et al., hep-ex/9807005.

[3] C. Athanassopoulos et al., Phys. Rev. Lett. 77 (1996) 3082, nucl-ex/9706006 and nuclex/9709006.

[4] B. Armbruster et al., Phys. Rev. C57 (1998) 3414 and G. Drexlin, talk at Wein'98.

[5] L. Wolfenstein, Phys. Rev. D17 (1978) 2369;

S.P. Mikheyev and A. Yu Smirnov, Sov. J. Nucl. Phys. 42 (1986) 913.

[6] G.L. Fogli, E. Lisi and D. Montanino, hep-ph/9709473 and hep-ph/9803309;

B. Faïd, G.L. Fogli, E. Lisi and D. Montanino, hep-ph/9805293;

J.N. Bahcall, P.I. Krastev and A. Yu Smirnov, hep-ph/9807216.

[7] M. Apollonio et al., Phys. Lett. B 420 (1998) 397.

[8] V. Barger, S. Pakvasa, T.J. Weiler and K. Whisnant, hep-ph/980638; V. Barger, T.J. Weiler and K. Whisnant, hep-ph/9807319.

[9] A.J. Baltz, A.S. Goldhaber and M. Goldhaber, hep-ph/9806540.

[10] R. Barbieri, L.J. Hall, D. Smith, A. Strumia and N. Weiner, hep/ph 9807235. 
[11] K.S. Babu and S.M. Barr, Phys. Lett. B 381 (1996) 202 (hep-ph/9511446).

[12] G. Costa and E. Lunghi, Nuovo Cim. A110 (1997) 549;

B. Brahmachari and R.N. Mohapatra, Phys. Rev. D58 (1998) (hep-ph/9710371);

S.F. King, hep-ph/9806440;

J.K. Elwood, N. Irges and P. Ramond, hep-ph/9807228;

Y. Nomura and T. Yanagida, hep-ph/9807325.

[13] B.C. Allanach, hep-ph/9806294.

[14] M. Jezabek and Y. Sumino, hep-ph/9807310.

[15] See for example L. K. Krauss, hep-ph/9807376. 